\title{
Initial Stress and Modified Ohm's Law in Magneto-thermoelastic Problem Under Three Theories with Microtemperatures and Voids
}

\author{
L. C. Bawankar*1, G. D. Kedar ${ }^{2}$ \\ ${ }^{1}$ Sanjivani College of Engineering, Kopargaon, Ahmednagar, India. \\ ${ }^{2}$ Department of Mathematics, RTM Nagpur University, Nagpur, India. \\ Email: ${ }^{1}$ latikabawankar@gmail.com
}

Received 7 May 2021, Revised 1 October 2021, Accepted 3 October 2021

\begin{abstract}
This paper studies the generalized magneto-thermoelastic problem with microtemperatures, voids taking into account initial stress and modified Ohm's law under three theories. The analytical solution is obtained by normal modes and expressions for micro temperature, temperature distribution, displacement, components of heat flux, change in the volume fraction field as well as stress components are calculated. The effect of initial stress and thermal shock is observed on desired field variables. The results are established graphically for all physical quantities and variation is done for three theories due to the effect of modified Ohm's law coefficient.
\end{abstract}

Keywords: Magneto-thermoelasticity; microtemperatures; voids; initial stress; modified Ohm's Law.

\section{Introduction}

The magneto-thermoelasticity theory tells us about the thermoelastic deformations in solids due to magnetic field presence, which is helpful in geophysics, plasma physics. The study of high temperatures and temperature gradient is vital with magnetic field presence to understand its effect on seismic waves and the emissions from nuclear devices in the form of electromagnetic radiations. For linear coupled and uncoupled thermoelasticity theory, diffusion type of heat conduction equations is considered, which predict infinite heat wave propagation speed contrary to physical observations. To eradicate the principle of coupled thermoelasticity, Biot [1] proposed the contradiction found in the classical uncoupled hypothesis that elastic changes do not influence on temperature. However, the hypothesis of both heat equations is of the kind of diffusion, estimating infinite heat wave propagation rates. The Lord and Shulman [2] was initiated by introducing a relaxation constant to account for the time required to accelerate the heat flow. If the constant of relaxation is set to zero, the equations of Lord and Shulman [2] decrease to classical field equation of thermoelasticity. The hyperbolic heat equation is consistent with this theory, removing the paradox of infinite heat wave propagation. The Green and Lindsay [3] named thermoelasticity as a temperature-dependent where its rating is included with two constant variables act as a relaxation time, which was not violating the classical Fourier's law. Agarwal [4] discussed the effect on plane thermoelastic waves due to presence of electromagnetic field. Paria [5] observed elastic and thermoelastic behavior of different problems under magnetic field influence. Youssef [6] studied the variable material properties in generalized thermoelasticity problems.

The theory of microtemperature is regarded as a theory that tackles temperature, wave propagation, and the thermal properties variation at microstructure level in a rigid thermocouple. The solid nanostructures are essential as particle can contract or stretch, and in solid vessels principal thermal stresses can be related to the effects of thermal microstructure, and so a well-structured theory of rigid solids that makes the effects of microtemperature is required. Grot [7] established the theory of microstructure in thermodynamic problem with inner structure. Microelements contain microtemperature, which causes microdeformations. Riha [8] applied micromorphic continua theory to heat conduction problem with inner structure and observed the changes in materials. Casas and Quintanilla [9] constructed the exponential stability of thermoplastic materials which have inner structure. Casas and Quintanilla and Iesan [10] discussed the microstructure of microelements in thermoelastic materials. Iesan [11] discussed the micromorphic elastic solids with microtemperatures. Scavanadze [12] provide the solution of equilibrium equation, constitutive relation with microtemperature by means of elementary function theory. Othman and Abd Elaziz [13] discussed gravity and microtemperature presence in a porous medium, and comparison is made for three theories. Quintanilla [14] have studied the uniqueness theorem of porous media with microtemperature under three theories of thermoelasticity. Eringen [15] and Eringen and Suhubi [16] discussed the micromorphic elastic solids and their behavior in the classical theory of thermoelasticity. Kalkal et al. [17] discussed the thermoelastic half space problem of microtemperature with diffusion under presence of magnetic field.

The voids are small pores distributed in elastic materials. It consists of volume and if it tends to zero becomes the limiting case for the classical theory of elasticity. The practical use of voids is important for investigating various types of biological and geological materials, but the elastic theory is not sufficient. The linear and nonlinear theory of 
elastic material with voids developed by Cowin and Nunziato [18], Nunziato and Cowin [19], Iesan [20]. The asymptotic spatial behaviour of material with voids in linear theory studied by Pompei and Scalia [21] and Stan Chirita [22]. Othman et al. [23] and Iesan [24] studied initial stress effect on thermoelastic material with voids. The concept of voids in a material distributed in the form granules introduced by Goodman and Cowin [25] and Jaric [26]. Othman et al. [27] developed the mathematical model of thermoelasticity with the existence of initial stress, voids, and microtemperature. Kalkal et al. [28] discussed wave nature in initially stressed thermoelastic problem with voids and microtemperature under magnetic field influence. Othman and Abd-Elaziz [29] observed effect of hall current and initial stress in porous thermoelastic condition with microtemperature. Othman and Abd-Elaziz [30] discussed about the rotation and hall effect on plane waves with voids and microtemperature on thermoelastic materials with magnetic field.

Initial stresses arise due to many reasons such as temperature variation, quenching process, living tissue growth and development, and gravity variations, etc. The study of initial stresses in thermal and mechanical conditions is critical as the earth consists of high initial stress due to gravity and strongly affects the propagation of waves. Ames and Straughan [31] proved the thermoelastic solids has continuous dependence if it is initially pre-stressed. The initial hydrostatic stress studied by Montanaro [32] in the linear theory of thermoelasticity. Abbas and Othman [33] investigated the interaction of thermoelastic half-space with initial hydrostatic stress under the fiber-reinforced anisotropic conditions.

The modified Ohm's law relates to the temperature gradient and current density. Lorentz force arises due to the interaction of magnetic, electric field and Ohm's law useful for the current density, which describes electric fieldinduced due to material particle velocity which moves in a magnetic field. Ezzat and Elall [34] and Sarkar [35] explained the problems of magneto-thermoelasticity by using modified Ohm's law and shows its effect on three different theories.

The novelty of this article is the introduction of modified Ohm's law in magneto-thermoelastic problem with microtemperature and voids under initial stress and thermal shock conditions for three theories. Modification in Ohm's law is due to the addition of temperature gradient term. This modification states that the electric potential gradient is proportional to the strength of the material at each point. The strength and temperature variation in a material at each point is observed because of microtemperature theory and modified Ohm's law.

In this work, we study the effect of modified Ohm's law with voids and microtemperature in the magnetothermoelastic problem under initial stress. The initially stressed linear, isotropic, homogeneous half-space problem under the influence of magnetic field is considered. The expressions for the desired variables are derived for Green Lindsay (GL), Lord and Shulman, and coupled theory (CT) for isothermal boundary conditions subjected to thermal shock by using normal mode analysis. Estimations have been carried out numerically and illustrated graphically for the above said theories due to the coefficient of modified Ohm's law.

\section{Basic Equations}

In the linear theory of thermodynamics, the governing equations for homogeneous isotropic magnetothermoelastic material with microtemperature, voids and initial stress can be written as

$\sigma_{i j}=2 \mu e_{i j}+\left(\lambda e_{r r}+\lambda_{0} \phi\right) \delta_{i j}-\beta\left(1+\tau_{1} T_{, t}\right) \delta_{i j}-$

$p\left(\delta_{i j}+\omega_{i j}\right)$

$h_{i}=\alpha \phi_{, i}-\mu_{1} w_{i}$

$g^{*}=-\lambda_{0} e_{r r}-\xi_{1} \phi+m T-w_{0} \phi_{, t}$

$q_{i}+\tau_{0} q_{i, t}=-k_{1} w_{i}-k^{*} T_{, i}$

$q_{i j}=-k_{4} w_{r, r} \delta_{i j}-k_{5} w_{i, j}-k_{6} w_{j, i}$

$Q_{i}=\left(k^{*}-k_{3}\right) T_{, i}+\left(k_{1}-k_{2}\right) w_{, i}$

$\rho \eta^{*}=a_{0} T+\beta e_{r r}+m \phi$

$\rho \varepsilon_{i}=-\mu_{1} \phi_{, i}-b w_{i}$

The rotation tensor and strain-displacement relation are

$e_{i j}=\frac{1}{2}\left(u_{i, j}+u_{j, i}\right), \omega_{i j}=\frac{1}{2}\left(u_{j, i}-u_{i, j}\right) i, j, r=1,2,3$

The equation of motion with Lorentz force be

$\sigma_{i j}+F_{i}=\rho u_{i, t t}$

$h_{i, i}+g^{*}=\rho \psi \phi_{, t t}$

The balance energy equation without heat source is

$-q_{i, i}=\rho T_{0} \eta_{, t}^{*}$

The first-moment of energy is

$\rho \varepsilon_{i, t}=-Q_{i}-q_{i}+q_{j i, j}$

where constants are defined in nomenclature.

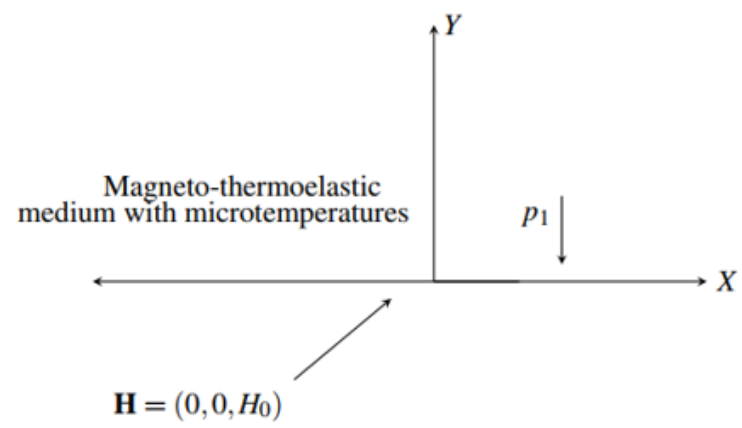

Figure 1: The geometry of the problem

\section{Problem Formulation}

Consider 2-D problem of isotropic, homogeneous electrically and thermally conducting thermoelastic half space $(y \geq 0)$ with voids and microtemperature. For twodimensional problem microtemperature vector is assumed as $w_{i}=\left(w_{1}, w_{2}, 0\right)$ and displacement vector $u_{i}=(u, v, 0)$. The constant magnetic field $\left(0,0, H_{0}\right)$ act normal to bounding plane (along positive $\mathrm{z}$-direction) and applied to the medium which produces $\boldsymbol{h}$ and $\boldsymbol{E}$ as given in Figure 1. The electromagnetic field equations for a conducting 
homogeneous medium which satisfy Maxwell's equations are

$\mathbf{J}+\varepsilon_{0} \dot{\mathbf{E}}=\nabla \times \mathbf{h}$

$\dot{\mathbf{B}}=\nabla \times \mathbf{E}$

$\operatorname{div} \mathbf{B}=\rho_{e}, \operatorname{div} \mathbf{D}=0$

$\boldsymbol{B}=\mu_{0}\left(\mathbf{H}_{\mathbf{0}}+\mathbf{h}\right), \mathbf{D}=\varepsilon_{0} \mathbf{E}$

In addition to this, in a finite conducting medium with modified Ohm's law is given by

$\mathbf{J}=\sigma_{0}\left(\mathbf{E}+\mu_{0} \dot{\mathbf{u}} \times \mathbf{H}\right)-\boldsymbol{k}_{\mathbf{0}} \nabla \mathbf{T}$

where constants are defined in nomenclature section.

Solving equations (14) to (18) we obtain

$h_{, x}=\sigma_{0}\left(E+\mu_{0} H_{0} \dot{u}\right)+k_{0} T_{, y}-\varepsilon_{0} \dot{E_{y}}$

$h_{, y}=\sigma_{0}\left(E+\mu_{0} H_{0} \dot{v}\right)-k_{0} T_{, x}+\varepsilon_{0} \dot{E}_{x}$

$\mu_{0} \dot{h}=E_{x, y}-E_{y, x}$

Lorentz force components from equation (17) and (18) are

$$
\begin{aligned}
& F_{x}=\mu_{0} H_{0} \sigma_{0}\left(E_{y}-\mu_{0} H_{0} \dot{u}\right)-k_{0} T_{, y} \\
& F_{y}=-\mu_{0} H_{0} \sigma_{0}\left(E_{x}+\mu_{0} H_{0} \dot{v}\right)+k_{0} T_{, x} \\
& F_{z}=0
\end{aligned}
$$

Using equations (1) to (9), (22) and (23) in equations (10) to (13), we obtain the linear partial differential equations with microtemperature, voids, modified Ohm's law under initial stress.

Equation of motion becomes

$\left(\mu-\frac{p}{2}\right) \nabla^{2} u+\lambda_{0} \phi_{, x}+\left(\lambda+\mu+\frac{p}{2}\right) e_{, x}-\beta(1+$

$\left.\tau_{1} \frac{\partial}{\partial t}\right) T_{, x}+\mu_{0} H_{0} \sigma_{0}\left(E_{y}-\mu_{0} H_{0} \dot{u}\right)-k_{0} T_{, y}=\rho \ddot{u}$

$\left(\mu-\frac{p}{2}\right) \nabla^{2} v+\lambda_{0} \phi_{, y}+\left(\lambda+\mu+\frac{p}{2}\right) e_{, y}-\beta(1+$

$\left.\tau_{1} \frac{\partial}{\partial t}\right) T_{, y}-\mu_{0} H_{0} \sigma_{0}\left(E_{x}+\mu_{0} H_{0} \dot{v}\right)+k_{0} T_{, x}=\rho \ddot{v}$

Volume fraction field equation is

$\left(\alpha \nabla^{2}-\xi_{1}-\rho \psi \frac{\partial^{2}}{\partial t^{2}}-w_{0} \frac{\partial}{\partial t}\right) \phi-\mu_{1} e_{1}-\lambda_{0} e+m(1+$

$\left.\tau_{1} \frac{\partial}{\partial t}\right) T=0$

$k^{*} T_{, x}=\left(1+\tau_{0} \frac{\partial}{\partial t}\right)\left[k_{6} \nabla^{2} w_{1}-\mu_{1} \dot{\phi_{, x}}+\left(k_{4}+k_{5}\right) e_{1, x}-\right.$

$\left.b \dot{w}_{1}-\left(k^{*}-k_{3}\right) T_{, x}+\left(k_{1}-k_{2}\right) w_{1}\right]-k_{1} w_{1}$

$k^{*} T_{, y}=\left(1+\tau_{0} \frac{\partial}{\partial t}\right)\left[k_{6} \nabla^{2} w_{2}-\mu_{1} \dot{\phi_{, y}}+\left(k_{4}+k_{5}\right) e_{1, y}-\right.$

$\left.b \dot{w}_{2}+\left(k^{*}-k_{3}\right) T_{, y}+\left(k_{1}-k_{2}\right) w_{2}\right]-k_{1} w_{2}$

Heat conduction equation is given by

$\left[k^{*} \nabla^{2}-a_{0} T_{0}\left(\frac{\partial}{\partial t}+\tau_{0} \frac{\partial^{2}}{\partial t^{2}}\right)\right] T+k_{1} e_{1}=\left(\frac{\partial}{\partial t}+\right.$

$\left.\eta_{0} \tau_{0} \frac{\partial^{2}}{\partial t^{2}}\right)\left(\beta T_{0} e+m T_{0} \phi\right)$ where $e=\frac{\partial u}{\partial x}+\frac{\partial v}{\partial y}, \quad e_{1}=\frac{\partial w_{1}}{\partial x}+\frac{\partial w_{2}}{\partial y}$

We introduce the following nondimensional variables

$\left(x_{i}^{\prime}, u_{i}^{\prime}\right)=\frac{\omega^{*}}{c_{1}}\left(x_{i}, u_{i}\right), \phi^{\prime}=\frac{\psi \omega^{* 2}}{c_{1}^{2}} \phi, w_{i}{ }^{\prime}=\frac{c_{1}}{\omega^{*}} w_{i}$,

$q_{i j}{ }^{\prime}=\frac{\omega^{*}}{\mu c_{1}^{2}} q_{i j}, \quad T^{\prime}=\frac{1}{\mu T_{0}} T, \quad \sigma_{i j}{ }^{\prime}=\frac{1}{\beta T_{0}} \sigma_{i j}$,

$h^{\prime}=\frac{\omega^{*}}{\mu_{0} H_{0} \sigma_{0}} h, \quad E_{i}{ }^{\prime}=\frac{\omega^{*} c_{1}}{\mu_{0}{ }^{2} H_{0} \sigma_{0}} E_{i}, \quad k_{0}{ }^{\prime}=\frac{\mu_{0} H_{0}}{\beta} k_{0}$

$\left(t^{\prime}, \tau_{0}{ }^{\prime}, \tau_{1}{ }^{\prime}\right)=\omega^{*}\left(t, \tau_{0}, \tau_{1}\right), p^{\prime}=\frac{1}{\beta T_{0}} p$,

$\omega^{*}=\frac{a_{0} T_{0} c_{1}^{2}}{k^{*}}, c_{1}^{2}=\frac{\lambda+2 \mu}{\rho}$

\section{Problem Solution}

Suppose that the potential functions in dimensionless form can be consider as $q_{1}(x, y, t)$, $q_{2}(x, y, t), N_{1}(x, y, t)$, and $N_{2}(x, y, t)$

$u=N_{1, x}+N_{2, x}, \quad v=N_{1, y}-N_{2, x}$,

$w_{1}=q_{1, x}+q_{2, y,} w_{2}=q_{1, y}-q_{2, x}$

To get the solution of dimensionless physical quantities, it is suitable to presume solution by using normal modes in the form

$\left[N_{1}, N_{2}, q_{1}, q_{2}, \emptyset, h, T\right](x, y, t)=$

$\left[N_{1}{ }^{*}, N_{2}{ }^{*}, q_{1}{ }^{*}, q_{2}{ }^{*}, \emptyset^{*}, h^{*}, T^{*}\right](y) e^{i(a x-\xi t)}$

Using equations (31) to (33) in equations (25) to (30) we obtained

$\left(D^{2}-L_{1}\right) h^{*}+L_{2}\left(D^{2}-a^{2}\right) N_{1}^{*}=0$

$\left(D^{2}-L_{3}\right) T^{*}+L_{4}\left(D^{2}-a^{2}\right) q_{1}{ }^{*}-L_{5}\left(D^{2}-a^{2}\right) N_{1}{ }^{*}-$

$L_{6} \emptyset^{*}=0$

$\left(D^{2}-L_{7}\right) \emptyset^{*}-m_{11}\left(D^{2}-a^{2}\right) N_{1}{ }^{*}-m_{12}\left(D^{2}-a^{2}\right) q_{1}{ }^{*}+$

$L_{8} T^{*}=0$

$\left(D^{2}-L_{9}\right) N_{1}{ }^{*}+L_{10} \emptyset^{*}-L_{11} T^{*}-L_{12} h^{*}=0$

$\left(D^{2}-L_{13}\right) N_{2}{ }^{*}+m_{6} T^{*}=0$

$\left(L_{18} D^{2}-L_{14}\right) q_{1}{ }^{*}+L_{16} \emptyset^{*}-L_{15} T^{*}=0$

$\left(m_{15} D^{2}-L_{17}\right) q_{2}{ }^{*}=0$

All the constants $m_{1}-m_{33}$ and $L_{1}-L_{18}$ are mentioned in Appendix and $D=d / d y$. By eliminating functions $N_{1}{ }^{*}, N_{2}{ }^{*}, q_{1}{ }^{*}, q_{2}{ }^{*}, \emptyset^{*}, h^{*}, T^{*}$ among equations (34) to (40) yields as follows

$\left(D^{12}-A D^{10}+B D^{8}-C D^{6}+E D^{4}-F D^{2}+\right.$

G) $\left(N_{1}{ }^{*}, N_{2}{ }^{*}, q_{1}{ }^{*}, q_{2}{ }^{*}, \emptyset^{*}, h^{*}, T^{*}\right)=0$

where $A, B, C, D, E, F, G$ are constants. Equation (41) can be classified as

$\left[\left(D^{2}-\propto_{1}^{2}\right)\left(D^{2}-\propto_{2}^{2}\right)\left(D^{2}-\propto_{3}^{2}\right)\left(D^{2}-\propto_{4}^{2}\right)\left(D^{2}-\propto_{5}^{2}\right)\left(D^{2}-\right.\right.$

$\left.\left.\propto_{6}^{2}\right)\right]\left(N_{1}{ }^{*}, N_{2}{ }^{*}, q_{1}{ }^{*}, q_{2}{ }^{*}, \emptyset^{*}, h^{*}, T^{*}\right)=0$ 
where $\propto_{n}^{2}(n=1,2,3,4,5,6)$ are the roots of characteristic equation. The general solution of physical quantities obtained from equation (41), bounds as $y \rightarrow \infty$ are

$\left(N_{1}{ }^{*}, N_{2}{ }^{*}, q_{1}{ }^{*}, q_{2}{ }^{*}, \emptyset^{*}, h^{*}, T^{*}\right)(y)=$

$\sum_{n=1}^{6}\left(1, O_{1_{n}}, O_{2_{n}}, O_{3_{n}}, O_{4_{n}}, O_{5_{n}}\right) S_{n} e^{-\alpha_{n} y+i(a x-\xi t)}$

where $S_{n}(n=1,2 \ldots 6)$ are constants. $O_{1_{n} \ldots . . .5_{n}}$ are some parameters depending on $a, \xi$ given in appendix.

The solution of equations (34) to (40) as $y \rightarrow \infty$ can be written as

$q_{2}(x, y, t)=S_{7} e^{-\alpha_{7} y+i(a x-\xi t)}$

$(u, v)(x, y, t)=\sum_{n=1}^{6}\left[O_{6_{n}}, O_{7_{n}}\right] S_{n} e^{-\alpha_{n} y+i(a x-\xi t)}$

$\left(w_{1}, w_{2}\right)(x, y, t)=\left[\sum_{n=1}^{6}\left(i a,-\propto_{n}\right) O_{5_{n}} S_{n} e^{-\propto_{n} y}-\right.$

$\left.\left(\propto_{7}, i a\right) S_{7} e^{-\propto_{7} y}\right] e^{i(a x-\xi t)}$

$\left(\sigma_{x x}, \sigma_{y y}, \sigma_{z z}\right)(x, y, t)=-p+$

$\sum_{n=1}^{6}\left[O_{8_{n}}, O_{9_{n}}, O_{9^{*}}\right] S_{n} e^{-\propto_{n} y+i(a x-\xi t)}$

$\left(\sigma_{x y}, \sigma_{y x}, \sigma_{x z}, \sigma_{y z}\right)(x, y, t)=$

$\sum_{n=1}^{6}\left[O_{10_{n}}, O_{10^{*}{ }_{n}}, 0,0\right] S_{n} e^{-\propto_{n} y+i(a x-\xi t)}$

$\left(q_{x x}, q_{y y}, q_{x y}\right)(x, y, t)=$

$\left[\sum_{n=1}^{6}\left[O_{11_{n}}, O_{12_{n}}, O_{13_{n}}\right] S_{n} e^{-\alpha_{n} y}+\right.$

$\left.\left(b_{9}, b_{10}, b_{11}\right) S_{7} e^{-\alpha_{7} y}\right] e^{i(a x-\xi t)}$

where $Q(a, \xi)$ is the parameter depends on $a, \xi$.

Now considering the electric and magnetic field intensities $h_{0}, E_{x 0}, E_{y 0}$ respectively. The nondimensional field equations satisfied by these variables are given by

$h_{0, y}=\varepsilon_{1} E_{x 0}^{\cdot}$

$h_{0, x}=\varepsilon_{1} E_{y 0}^{\cdot}$

$h_{0}=\left(E_{x 0}\right)_{, y}-\left(E_{y 0}\right)_{, x}$

$h_{0}, E_{x 0}$, and $E_{y 0}$ can be decompose in normal modes in the following form

$\left[h_{0}, E_{x 0}, E_{y 0}\right](x, y, t)=\left[h_{0}{ }^{*}, E_{x 0}{ }^{*}, E_{y 0}{ }^{*}\right](y) e^{i(a x-\xi t)}$

Using Equation (53) to Equations (50) to (52) and after solving, the results are obtained for $y \rightarrow \infty$ as

$h_{0}{ }^{*}=Q(a, \xi) e^{-\alpha_{9} y}$

$E_{x 0}^{*}=\frac{\alpha_{9}}{\left(i \varepsilon_{1} \xi\right)} Q(a, \xi) e^{-\alpha_{9} y}$

$E_{y 0}^{*}=\frac{\alpha_{9}}{\left(i \varepsilon_{1} \xi\right)} Q(a, \xi) e^{-\alpha_{9} y}$

\section{Application}

To determine the parameter $S_{n}$ and $Q$ we need to consider the following boundary condition at $y=0$

1. Thermal boundary condition: The surface $y=0$ is exposed to time dependent thermal shock in the form

$$
T(x, 0, t)=f(x, t)
$$

2. Mechanical boundary condition:

a. The surface of the half space is stressed by constant force $p_{1}$ i.e.

$$
\sigma_{y y}(x, 0, t)=-p_{1} e^{i(a x-\xi t)}-p
$$

b. The surface of the half space is traction free

$$
\sigma_{x y}(x, 0, t)=0
$$

3. Electric Boundary condition: For $y=0$, the component of electric field intensity vector are continuous across the half surface

$$
E_{y}(x, 0, t)=E_{y 0}(x, 0, t)
$$

4. Magnetic Boundary condition: For $y=0$ the component of magnetic field intensity vector are continuous across the half surface

$$
h(x, 0, t)=h_{0}(x, 0, t)
$$

5. Heat flux moments Boundary condition: The heat flux moments along normal and tangential direction are free.

$$
q_{x x}(x, 0, t)=q_{x y}(x, 0, t)=0
$$

6. Volume fraction field boundary condition: The condition of volume fraction field change is

$$
\frac{\partial \phi}{\partial y}=0
$$

Substituting the desired physical quantities into the above boundary conditions and finding the value of constants by using matrix inversion method we get

$\left[\begin{array}{l}S_{1} \\ S_{2} \\ S_{3} \\ S_{4} \\ S_{5} \\ S_{6} \\ S_{7} \\ Q\end{array}\right]=\left[\begin{array}{cccccccc}O_{9_{1}} & O_{9_{2}} & O_{9_{3}} & O_{9_{4}} & O_{9_{5}} & O_{9_{6}} & 0 & 0 \\ O_{10_{1}} & O_{10_{2}} & O_{10_{3}} & O_{10_{4}} & O_{10_{5}} & O_{10_{6}} & 0 & 0 \\ O_{1_{1}} & O_{1_{2}} & O_{1_{3}} & O_{1_{4}} & O_{1_{5}} & O_{1_{6}} & 0 & 0 \\ O_{4_{1}} & O_{4_{2}} & O_{4_{3}} & O_{4_{4}} & O_{4_{5}} & O_{4_{6}} & 0 & 1 \\ O_{14_{1}} & O_{14_{2}} & O_{14_{3}} & O_{14_{4}} & O_{14_{5}} & O_{14_{6}} & 0 & -L \\ O_{11_{1}} & O_{11_{2}} & O_{11_{3}} & O_{11_{4}} & O_{11_{5}} & O_{11_{6}} & b_{10} & 0 \\ O_{12_{1}} & O_{12_{2}} & O_{123} & O_{12_{4}} & O_{12_{5}} & O_{12_{6}} & b_{11} & 0 \\ \alpha_{1} O_{2_{1}} & \alpha_{2} O_{22} & \alpha_{3} O_{23} & \alpha_{4} O_{24} & \alpha_{5} O_{25} & \alpha_{6} O_{26} & 0 & 0\end{array}\right]^{-1}\left[\begin{array}{c}-p_{1} \\ 0 \\ f^{*} \\ 0 \\ 0 \\ 0 \\ 0 \\ 0\end{array}\right]$

\section{Perticular and Special cases}

6.1 Initially stressed thermoelastic problem with voids and microtemperature by neglecting magnetic field

By neglecting the magnetic field $H_{0}=0, k_{0}=0$ in the governing equation we obtained the equation for initially stressed thermoelastic half space problem with microtemperature and voids are given by

$\left[D^{2}-L_{3}\right] T^{*}+L_{4}\left[D^{2}-a^{2}\right] q_{1}^{*}-L_{5}\left[D^{2}-a^{2}\right] N_{1}^{*}-L_{6} \emptyset^{*}=$ 0

$\left[D^{2}-L_{7}\right] \emptyset^{*}-m_{11}\left[D^{2}-a^{2}\right] N_{1}^{*}-m_{12}\left[D^{2}-a^{2}\right] q_{1}^{*}+$

$L_{8} T^{*}=0$

$\left[D^{2}-L_{9}\right] N_{1}^{*}+L_{10} \emptyset^{*}-L_{11} T^{*}=0$

$\left[D^{2}-L_{13}\right] N_{2}^{*}=0$ 
$\left[m_{20} D^{2}-L_{14}\right] q_{1}^{*}-L_{15} T^{*}+L_{16} \emptyset^{*}=0$

$\left[m_{15} D^{2}-L_{16}\right] q_{2}^{*}=0$

Eliminating functions $\emptyset^{*}, T^{*}, q_{1}^{*}, N_{1}^{*}, N_{2}^{*}, q_{2}^{*}$ from equations (64) to (69) we get

$$
\left(D^{8}-A_{1} D^{6}+A_{2} D^{4}-A_{3} D^{2}+A_{4}\right)\left(N_{1}^{*}, N_{2}^{*}, \emptyset^{*}, q_{1}^{*}, T^{*}\right)=
$$

0

where $A_{1}, A_{2}, A_{3}$, and $A_{4}$ are constants. The general solution of physical quantities obtained from equation (70), bounds as $y \rightarrow \infty$ are

$\left(T^{*}, \emptyset^{*}, N_{1}^{*}, q_{1}^{*}\right)(y)=$

$\sum_{n=1}^{4}\left(1, H_{1 n}, H_{2 n}, H_{3 n}\right) S_{n}^{*} e^{-\alpha_{n}^{*} y+i(a x-\xi t)}$

The solution of equations (64) to (69) as $y \rightarrow \infty$ can be written as

$$
\begin{aligned}
& N_{2}(x, y, t)=S_{5}^{*} e^{-\alpha_{6} y+i(a x-\xi t)} \\
& q_{2}(x, y, t)=S_{6}^{*} e^{-\alpha_{7}^{*} y+i(a x-\xi t)} \\
& (\mathrm{u}, \mathrm{v})(x, y, t)=\sum_{n=1}^{4}\left(i a,-\alpha_{n}^{*}\right) H_{2} S_{n}^{*} e^{-\alpha_{n}^{*} y+i(a x-\xi t)}- \\
& \left(\alpha_{5}^{*}, i a\right) S_{5} e^{-\alpha_{5}^{*} y+i(a x-\xi t)} \\
& \left(w_{1}, w_{2}\right)(x, y, t)=\sum_{n=1}^{4}\left(i a,-\alpha_{n}^{*}\right) H_{3 n} S_{n}^{*} e^{-\alpha_{n}^{*} y+i(a x-\xi t)}- \\
& \left(\alpha_{6}^{*}, i a\right) S_{6}^{*} e^{-\alpha_{6}^{*} y+i(a x-\xi t)} \\
& \left(\sigma_{x x}, \sigma_{y y}\right)(x, y, t)=-p+ \\
& \sum_{n=1}^{4}\left(H_{4 n}, H_{5 n}\right) S_{n}^{*} e^{-\alpha_{n}^{*} y+i(a x-\xi t)}+ \\
& \left(Z_{1}, Z_{2}\right) S_{5}^{*} e^{-\alpha_{5}^{*} y+i(a x-\xi t)} \\
& \sigma_{x y}(x, y, t)=\sum_{n=1}^{4} H_{6_{n}} S_{n}^{*} e^{-\alpha_{n}^{*} y+i(a x-\xi t)}+ \\
& Z_{3} S_{5}^{*} e^{-\alpha_{5}^{*} y+i(a x-\xi t)} \\
& \left(q_{x x}, q_{y y}\right)(x, y, t)=\sum_{n=1}^{4}\left(H_{7 n}, H_{8_{n}}\right) S_{n}^{*} e^{-\alpha_{n}^{*} y+i(a x-\xi t)}+ \\
& \left(Z_{4}, Z_{5}\right) S_{6}^{*} e^{-\alpha_{6}^{*} y+i(a x-\xi t)}
\end{aligned}
$$

where $\alpha_{n}^{*}, \alpha_{5}^{*}$, and $\alpha_{6}^{*}$ are the roots of characteristic equation, $S_{n}$ are constants.

$H_{1_{n} \ldots \ldots \ldots . .3_{n}}$ are some parameters depending on $a$, $\xi$ given in Appendix. Substituting the all above physical quantities into the equations (57) to (59), (62) and (63) and finding the value of constants by using matrix inversion method we get

$$
\left[\begin{array}{l}
S_{1}^{*} \\
S_{2}^{*} \\
S_{3}^{*} \\
S_{4}^{*} \\
S_{5}^{*} \\
S_{6}^{*}
\end{array}\right]=\left[\begin{array}{cccccc}
H_{5_{1}} & H_{5_{2}} & H_{5_{3}} & H_{5_{4}} & Z_{2} & 0 \\
H_{6_{1}} & H_{6_{2}} & H_{6_{3}} & H_{6_{4}} & Z_{3} & 0 \\
1 & 1 & 1 & 1 & 0 & 0 \\
H_{7_{1}} & H_{7_{2}} & H_{7_{3}} & H_{7_{4}} & 0 & Z_{4} \\
H_{8_{1}} & H_{8_{2}} & H_{8_{3}} & H_{8_{4}} & 0 & Z_{5} \\
H_{1_{1}} & H_{1_{2}} & H_{1_{3}} & H_{1_{4}} & 0 & 0
\end{array}\right]^{-1}\left[\begin{array}{c}
-p_{1} \\
0 \\
f^{*} \\
0 \\
0 \\
0
\end{array}\right]
$$

The solution and graphical analysis match with the Othman et.al.[28]
6.2 Magneto-thermoelastic problem with modified Ohm's law by neglecting voids, initial stress and microtemperature

To study this problem we consider all parameters of voids $\left(\alpha=\psi=\xi_{1}=\omega_{0}=\lambda_{0}=m=0\right)$, initial stress $(p=0)$ and microtemperature $\left(k_{1}=k_{2}=k_{3}=k_{4}=k_{5}=\right.$ $\left.k_{6}=b=0\right)$ as zero. After finding solution and graphical analysis this model outcomes match with those of Sarkar [35].

6.3 Initially stressed magneto-thermoelastic problem with microtemperature by neglecting voids and modified Ohm's law

To study this problem, we consider all parameters of voids are taken as zero $\left(\alpha=\psi=\xi_{1}=\omega_{0}=\lambda_{0}=m=0\right)$. The solution for this problem and graphical analysis are done. The outcomes of this problem coincide with those of Kalkal et al. [18] (neglecting diffusion).

\section{Results and discussions}

For the numerical calculations magnesium material is chosen to illustrate the analytical results. According to Othman and Abd-Elaziz [29] the material constants are as follows

$\lambda=9.4 \times 10^{10} \frac{\mathrm{N}}{\mathrm{m}^{2}}, H_{0}=\frac{10^{7}}{4 \pi}, \beta=7.779 \times$

$10^{-8} \frac{\mathrm{N}}{\mathrm{m}^{2}}, \quad a_{0} T_{0}=1.8 \times 10^{6} \mathrm{Jm}^{-3} \mathrm{deg}^{-1}, \mu=4 \times$

$10^{10} \frac{\mathrm{N}}{\mathrm{m}^{2}}, k_{1}=3.5 \times 10^{-6} \frac{\mathrm{N}}{\mathrm{s}}, k_{2}=4.5 \times 10^{-6} \frac{\mathrm{N}}{\mathrm{s}}, p=$

$10, b=0.15 \times 10^{-9} N, k_{3}=5.5 \times 10^{-6} \frac{N}{s}, k_{4}=6.5 \times$

$10^{-6} \frac{N}{s m^{2}}, k_{5}=7.6 \times 10^{-6} \frac{N}{s m^{2}}, k_{6}=9.6 \times$

$10^{-6} \frac{N}{s m^{2}}, \mu_{1}=8.5 \times 10^{-6} N, \epsilon_{0}=\frac{10^{-9}}{36 \pi}, \mu_{0}=4 \pi \times$

$10^{-7}, \sigma_{0}=9.36 \times 10^{-5}, \mu=4 \times 10^{10} \frac{\mathrm{N}}{\mathrm{m}^{2}}, a=1.6 \mathrm{~m}, \chi_{0}=$

$0.02 \frac{\mathrm{rad}}{\mathrm{s}}, \eta_{0}=0.1, p_{1}=0.1 K, B=4, \tau_{0}=0,0.3, \tau_{1}=$

$0,0.1, \varsigma=1, \alpha_{t}=7.4033 \times 10^{-7} K^{-1}, T_{0}=298 K, k^{*}=$

$1.7 \times 10^{2} \frac{\mathrm{N}}{S K^{\prime}}$

Parameters of voids are considered from Iesan [20]

$\alpha=3.688 \times 10^{-5} N, \psi=1.753 \times 10^{-15} \mathrm{~m}^{2}$,

$\xi_{1}=1.475 \times 10^{10} \frac{N}{m^{2}}, \omega_{0}=0.787 \times 10^{-3} \frac{N}{m^{2} s}$,

$\lambda_{0}=1.1333 \times 10^{10} \frac{N}{m^{2}}, m=2 \times 10^{6} \frac{N}{\operatorname{deg} m^{2}}$

The function $f(x, t)=\theta_{0} H(|B|-x) e^{-\varsigma t}$, where $\theta_{0}$ is constant, $H(|B|-x)$ is the displayed Heaviside unit step function represents that around the $\mathrm{X}$-axis heat is applied with $2 \mathrm{~B}$ to keep the temperature $\theta_{0}$ although it is at zero on the rest of surface.

By using equation (33) to $f(x, t)$ we obtain

$$
f^{*}=\frac{\sqrt{2} \theta_{0} \sin (a B)(1+i a \pi \delta(a))}{\sqrt{\pi} a(\xi+\varsigma)}
$$


We consider $\xi=\chi_{0}+i \chi_{1}$ but for small time $\xi=\chi_{0}$. The calculations are done and the results presented in graphical form. The graphs are plotted for fixed value of time and space variables $t=0.1$ and $x=0.1$. The physical quantities are compared for presence and absence of coefficient of Ohm's law i.e. $k_{0}=0,0.1$ under coupled (CT), Lord Shulman (LS) (one relaxation time), Green Lindsay (GL) (two relaxation time) theory. Graphs are plotted for dimensionless field quantities. In Figures 2 to 9 the solid line represents CT theory results, the large dash line represents LS theory results and small dash line represents GL theory results. Figure 2 indicates the distribution of dimensionless temperature varies with $\mathrm{y}$. Value of temperature is large for GL theory in comparison to LS and GL theory. Temperature increase in the interval $0 \leq y \leq 1.8$ and then decrease gradually in the interval $1.8 \leq y \leq 6$. Figure 3 investigates the dimensionless displacement variations of $\mathrm{u}$ with $y$ values. The value of solutions for three theories is large for $k_{0}=0.1$ as compared to $k_{0}=0$. Maximum value of $\mathrm{u}$ appears in the range $0 \leq y \leq 1$ and small in the range $1.5 \leq y \leq 5.8$. Figure 4 shows dimensionless change in volume fraction field $\phi$ with the passage of distance. It shows significant change for $k_{0}=0,0.1$ in GL theory. GL theory attains maximum value in range $0 \leq y \leq 1.8$ for $k_{0}=0.1$ and $0 \leq y \leq 1$ for $k_{0}=0$. Figure 5 depicts variation of dimensionless microtemperature with the distance y. The graph shows increasing and decreasing effect on the magnitude of $w_{1}$.

Figure 6 describes the distribution of dimensionless normal stress $\sigma_{x x}$ with the $y$ values. The value of $\sigma_{x x}$ is maximum for GL theory, moderate for LS theory and minimum for CT theory. The graph is increasing for the distance $0 \leq y \leq 0.5$ and then gradually decreases and become constant for all three theories at $y \geq 6$. Figure 7 shows distribution of dimensionless tangential stress $\sigma_{x y}$ with distance. The value decrease in the interval $0 \leq y \leq$ 1 gradually increases for $1 \leq y \leq 6$. Figure 8 exhibits the distribution of dimensionless heat flux moment $q_{x x}$ for different values of coefficient of modified Ohm's law $k_{0}$. It shows slight variations in figures for $k_{0}=0,0.1$ in all theories. All the curves start with the zero which satisfies boundary conditions. Figure 9 represent dimensionless heat flux moment distribution of $q_{x y}$ with distance $y$. Curve attains maximum value for the range $1.3 \leq y \leq 6$ then decreases gradually and value becomes constant for $y \geq 7$.

Figures 10 and 11 represents dimensionless temperature and displacement distribution for three different cases. Variations of graphs are observed by considering particular cases that are initially stressed thermoelastic problem with voids and microtemperature (ITVM), magneto-thermoelastic problem with modified Ohm's law (MTM) and initially stressed magneto-thermoelastic problem with microtemperature (IMTM). Figure 10 shows dimensionless temperature gradually decreases for the range $0 \leq y \leq 4$ and becomes finite for all three cases. Figure 11 represents dimensionless displacement decreases initially in the interval $0 \leq y \leq 3$ and gradually increases to become finite. The boundary condition shows the response in a bounded region of space with the finite speed of propagation. Hence the significant change is observed under three theories by using modified Ohm's law $k_{0}=0.1$ as compared to absence i.e. $k_{0}=0$.

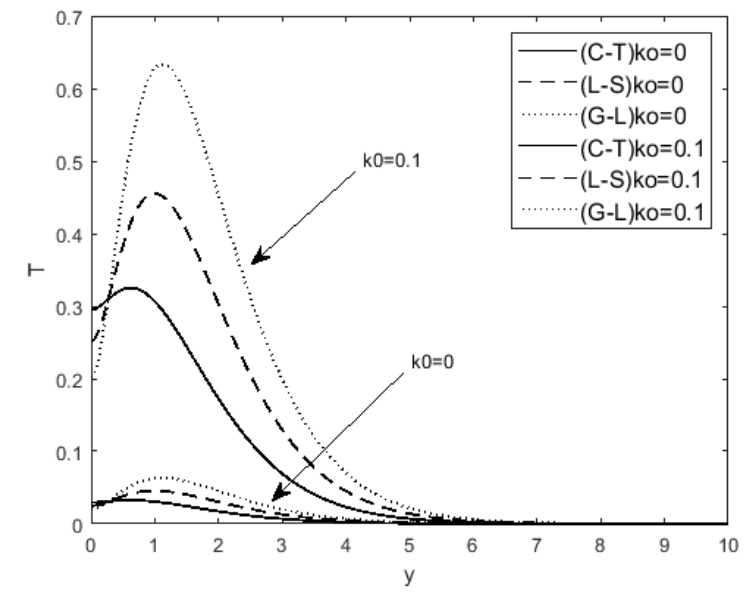

Figure 2: Temperature T variation for distinct values of $k_{0}$

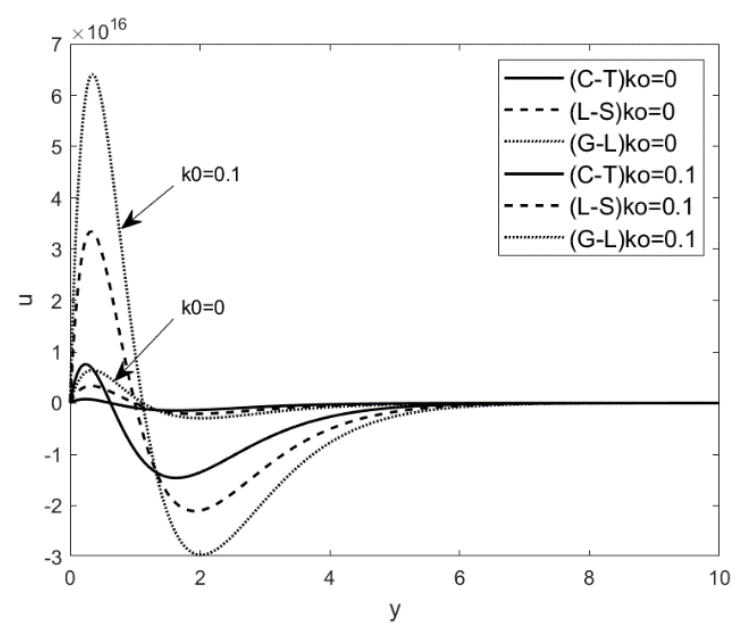

Figure 3: Displacement $u$ variation for distinct values of $k_{0}$

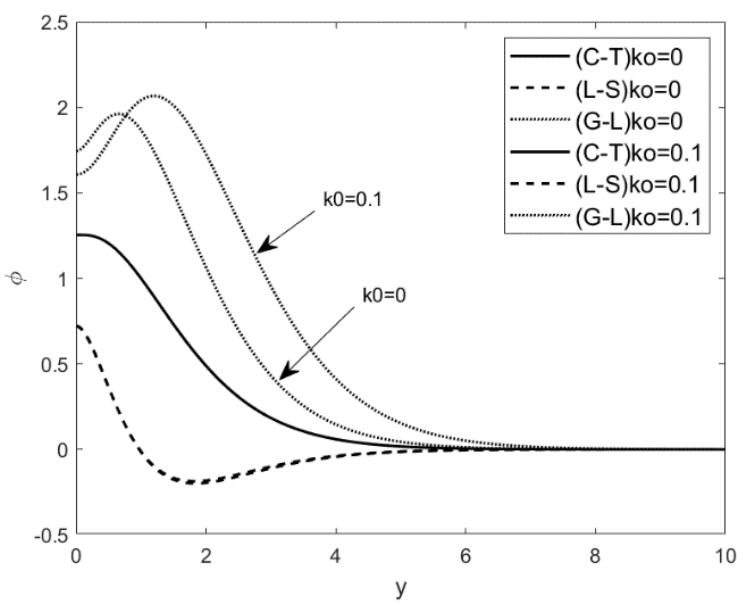

Figure 4: Change in volume field $\phi$ variation for distinct values of $k_{0}$ 


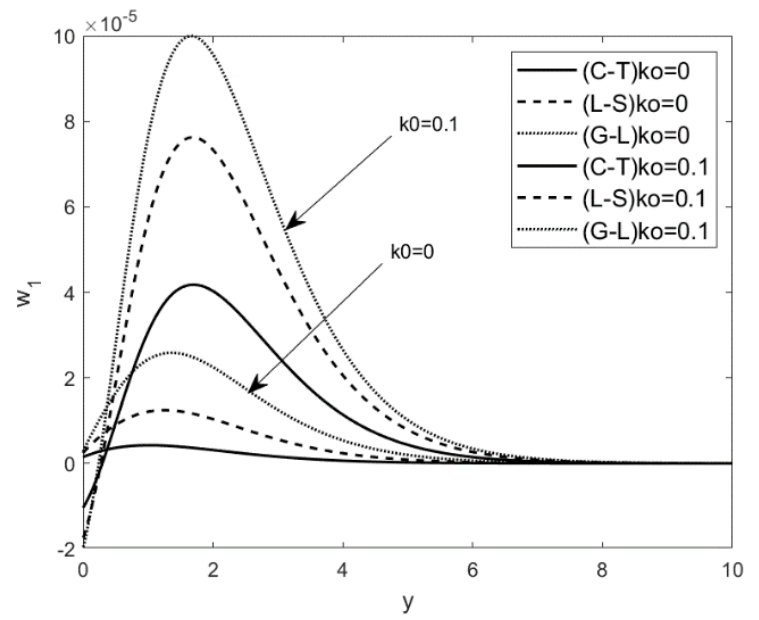

Figure 5: Microtemperature $w_{1}$ variation for distinct values of $k_{0}$

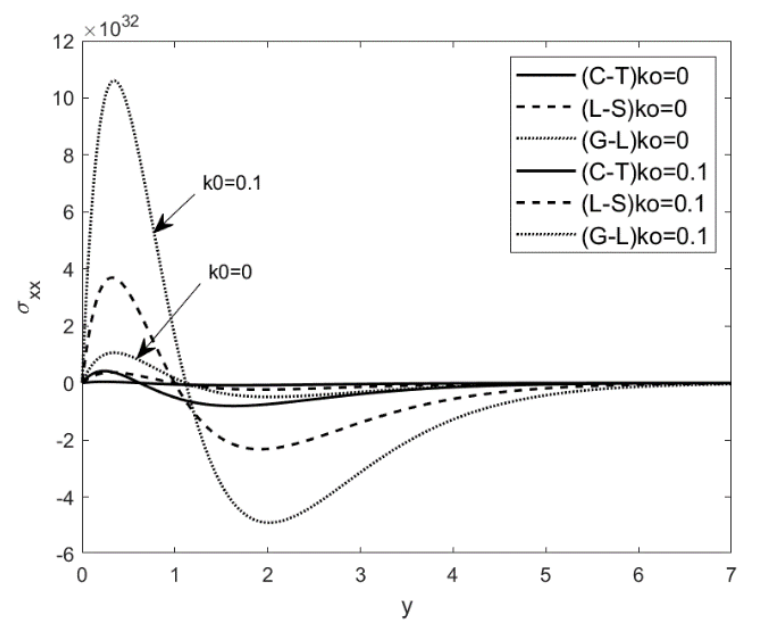

Figure 6: Normal stress $\sigma_{x x}$ variation for distinct values of $k_{0}$

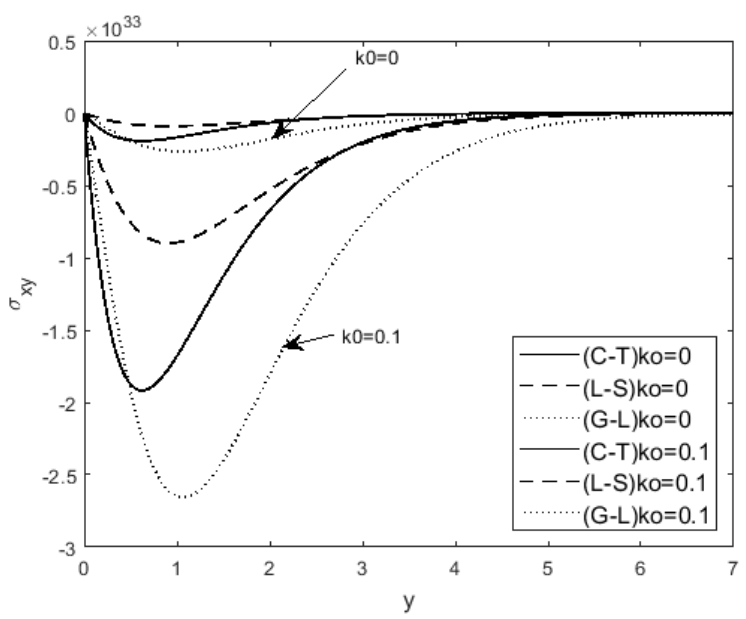

Figure 7: Shearing stress $\sigma_{x y}$ variation for distinct values of $k_{0}$

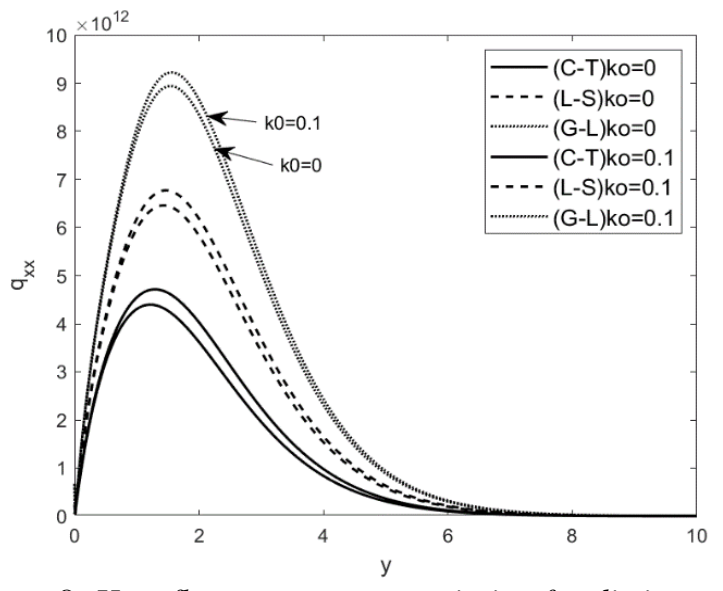

Figure 8: Heat flux moment $q_{x x}$ variation for distinct values of $k_{0}$

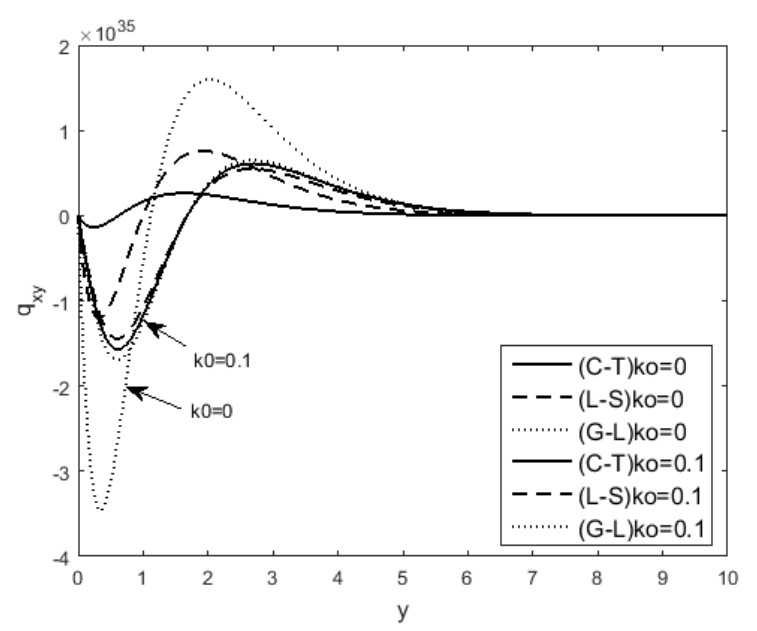

Figure 9: Heat flux moment $q_{x y}$ variation for distinct values of $k_{0}$

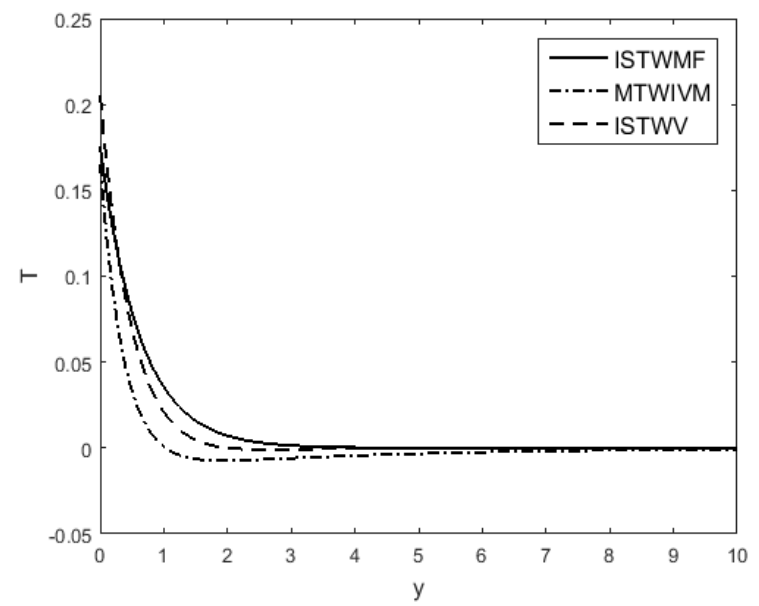

Figure 10: Temperature distribution $T$ with distance $y$ 


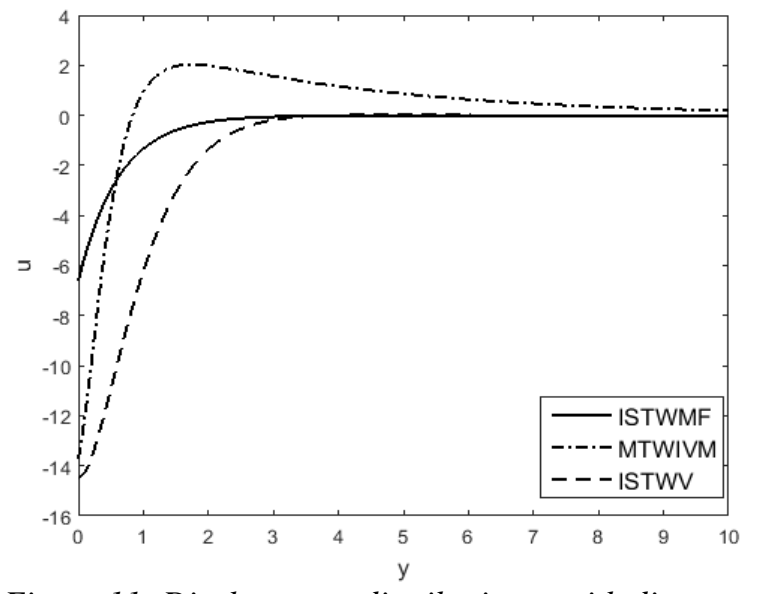

Figure 11: Displacement distribution $u$ with distance $y$

\section{Conclusion}

In this article, we present a model of magnetothermoelastic problem with modified Ohm's law, initial stress, voids, and microtemperatures in the context of GL, LS, and coupled theories. The problem is solved by using normal modes and effects of coefficient of modified Ohm's law on physical quantities are observed. The boundary conditions with thermal shock and initial stress is considered. From the graphical illustration we conclude the following facts which is useful to design new material in the development of the theory of magneto-thermoelasticity.

1. The relaxation time presence for the GL, LS leads to reduction of profile of temperature, volume fraction field, displacement, microtemperature, stresses along normal, moment of heat flux and increase in shear stresses with the change in coefficient of Ohm's law.

2. The increase in temperature values can be explained by the heat loss produced by the motion of electric current, which can be the main reason that the medium deformation tends to be normal.

3. The significant effect of modified ohm's law is observed with the change under three theories on all dimensionless field variables.

4. Finite speed of propagation is observed in all figures.

The microtemperature theory is useful to researcher's working on the area of drilling, mining tremors into the earth crust such as earthquake engineering, geophysics and seismologist. Because of the inclusion of modified Ohm's law in magneto-thermoelastic problem with microtemperature gives the new and novel contribution to this field.

\section{Acknowledgements}

The authors appreciate and thanks the anonymous reviewers for their valuable suggestions, which resulted in revising the paper to its present form.

\section{Nomenclature}

$H_{0} \quad$ Component of initial magnetic field vector

$Q_{i} \quad$ Mean heat flux moment component

$T$ Absolute temperature

$T_{0} \quad$ Reference temperature taken as $\left|\left(T-T_{0}\right) / T_{0}\right|<1$

$\alpha, \psi, \xi_{1}, m, \lambda_{0}, \omega_{0} \quad$ Material constant for voids

$\alpha_{t} \quad$ Coefficient of linear thermal expansion

$\beta=(3 \lambda+2 \mu) \alpha_{t}$
B Magnetic field induction vector

D Electric displacement vector

E Induced electric field vector

$\mathbf{F}_{\mathbf{i}} \quad$ Lorentz force

H Magnetic Intensity vector

J Current density vector

h Induced magnetic field vector

$\delta_{i j} \quad$ Kronecker delta function

$\epsilon_{0} \quad$ Electric Permeability

$\epsilon_{i} \quad$ First moment energy vector components

$\eta \quad$ Entropy per unit mass

$\lambda, \mu$ Lame's Constant

$\mu_{0} \quad$ Magnetic Permeability

$\phi \quad$ Change in volume fraction field

$\rho$ Density

$\sigma_{0} \quad$ Electric conductivity

$\sigma_{i j}$ Components of stress tensor

$\tau_{0}, \tau_{1}$ Relaxation times

$a_{0}, b, \mu_{1}, k_{i}(i=1,2,3,4,6)$ Coefficient of constitutive relations

$c_{1}^{2}=\frac{\lambda+2 \mu}{\rho}$ Speed of propagation of elastic waves

$g^{*} \quad$ Intrinsic equilibrated body force

$h_{i} \quad$ Equilibrated stress vector

$k^{*} \quad$ Thermal conductivity

$k_{0} \quad$ Modified Ohm's law coefficient

$p$ Pressure

$q_{i} \quad$ Heat flux vector components

$q_{i j}$ First heat flux moment vector components

$t$ Time variable

$u_{i}$ Displacement vector

$w_{i} \quad$ Microtemperature vector

\section{Appendix}

$m_{1}=\frac{\left(\lambda+\mu+\frac{\beta T_{0} p}{2}\right)}{\left(\mu-\frac{\beta T_{0} p}{2}\right)}, m_{2}=\frac{\left(\lambda_{0} c_{1}^{2}\right)}{\omega^{*^{2}} \psi\left(\mu-\frac{\beta T_{0} p}{2}\right)}, \quad m_{3}=\frac{\left(\beta T_{0}\right)}{\left(\mu-\frac{\beta T_{0} p}{2}\right)}$,

$m_{4}=\frac{\left(\mu_{0}^{3} \sigma_{0}^{2} H_{0}^{2}\right)}{\omega^{*^{2}}\left(\mu-\frac{\beta T_{0} p}{2}\right)}, m_{5}=\frac{\left(\sigma_{0} \mu_{0}^{2} H_{0}^{2}\right)}{\omega^{*^{2}}\left(\mu-\frac{\beta T_{0} p}{2}\right)}$

$m_{6}=\frac{\left(\beta k_{0} T_{0}\right)}{\mu_{0} H_{0}\left(\mu-\beta T_{0} p / 2\right)}, m_{7}=\frac{\left(\rho c_{1}^{2}\right)}{\left(\mu-\beta T_{0} p / 2\right)}, m_{8}=\frac{\rho c_{1}^{2} \psi}{\alpha}$,

$m_{9}=\frac{\omega_{0} c_{1}^{2}}{\alpha \omega^{*}}, m_{10}=\frac{\xi_{1} c_{1}^{2}}{\alpha \omega^{*}}, m_{11}=\lambda_{0} \psi / \alpha, m_{12}=\frac{\mu_{1} \omega^{*^{2}} \psi}{\alpha c_{1}^{2}}$,

$m_{13}=\frac{\left(m T_{0} \psi\right)}{\alpha}, m_{14}=k^{*} T_{0}, m_{15}=\frac{\left(k_{6} \omega^{*}\right)}{c_{1}}$,

$m_{16}=\frac{\left(\left(k_{4}+k_{5}\right) \omega^{*^{2}}\right)}{c_{1}^{2}}, \quad m_{17}=\left(k_{1}-k_{2}\right), \quad m_{18}=\left(k^{*}-k_{3}\right) T_{0}$,

$m_{19}=\frac{\mu_{1} c_{1}^{2}}{\psi \omega^{*}}, m_{20}=m_{15}-m_{16}, m_{21}=\frac{k^{*} \omega^{* 2}}{c_{1}^{2}}$,

$m_{22}=\frac{k_{1} \omega^{* 2}}{T_{0} c_{1}^{2}}, m_{23}=a_{0} T_{0} \omega^{*}, m_{24}=\beta \omega^{*}, \quad m_{25}=\frac{m c_{1}^{2}}{\psi \omega^{*}}$,

$m_{26}=\frac{c_{1}^{2}}{c_{0}^{2}}, m_{27}=\frac{\sigma_{0} \mu_{0} c_{1}^{2}}{\omega^{*}}, m_{28}=\frac{(\lambda+2 \mu) \omega^{*^{2}}}{\left(\beta T_{0} c_{1}^{2}\right)}, m_{29}=\frac{(\lambda+2 \mu)}{\left(\beta T_{0}\right)}$,

$m_{30}=\frac{(\lambda)}{\left(\beta T_{0}\right)}, m_{31}=\frac{\left(\lambda_{0} c_{1}^{2}\right)}{\left(\beta T_{0} \psi \omega^{*^{2}}\right)}, m_{32}=\left(1-\tau_{1} i \xi\right)$,

$m_{33}=\frac{\mu}{\left(\beta T_{0}\right)}, \quad m_{34}=b \omega^{*}, \quad c_{0}^{2}=\frac{1}{\mu_{0} \epsilon_{0}}, L=\frac{(a)}{\left(\xi \epsilon_{0}\right)}, \quad L_{1}=a^{2}-$

$m_{26} \xi^{2}-i \xi m_{27}, L_{2}=i \xi c_{1}^{2}, L_{3}=a^{2}-\frac{m_{23}\left(i \xi+\tau_{0} \xi^{2}\right)}{m_{21}}$, 


$$
\begin{aligned}
& L_{4}=\frac{m_{22}}{m_{21}}, L_{5}=\frac{m_{24}\left(i \xi+\eta_{0} \tau_{0} \xi^{2}\right)}{m_{21}}, L_{6}=\frac{m_{25}\left(i \xi+\eta_{0} \tau_{0} \xi^{2}\right)}{m_{21}}, \\
& \alpha_{7}=\sqrt{\frac{L_{17}}{m_{15}}}, L_{7}=a^{2}-m_{8} \xi^{2}-i \xi m_{9}+m_{10}, \\
& L_{8}=m_{13}\left(1-i \xi \tau_{1}\right), L_{9}=a^{2}-\frac{\left(i m_{5} \xi+m_{7} \xi^{2}\right)}{\left(1+m_{1}\right)}, L_{10}=\frac{\left(m_{2}\right)}{\left(1+m_{1}\right)} \\
& L_{11}=\frac{m_{3}\left(1-i \xi \tau_{1}\right)}{\left(1+m_{1}\right)}, \quad L_{12}=\frac{i c_{1}^{2} m_{4} \xi}{a^{2}\left(1+m_{1}\right)}, \quad L_{13}=a^{2}-m_{7} \xi^{2}- \\
& i \xi m_{5}, \quad L_{14}=\frac{m_{20} a^{2}+i m_{34} \xi-m_{17}+k_{1}}{\left(1+m_{1}\right)}, \quad L_{15}=m_{18}+\frac{m_{14}}{\left(1-i \xi \tau_{0}\right)} \\
& L_{16}=i \xi m_{19}, \quad L_{17}=\frac{k_{1}}{\left(1-i \xi \tau_{0}\right) m_{15}}, L_{18}=a^{2}-m_{17}-i \xi m_{20} \text {, } \\
& b_{6}=\frac{\omega^{*^{3}}}{\mu c_{1}^{4}}, \quad b_{7}=k_{4} b_{6}, \quad b_{8}=\left(k_{5}+k_{6}\right) b_{6}, \quad b_{9}=i a\left(b_{8}-\right. \\
& \left.b_{7}\right) \alpha_{7}, b_{10}=-i a b_{9} \alpha_{7}, b_{11}=b_{6}\left(\alpha_{7}^{2} k_{5}+a^{2} k_{6}\right) \text {, } \\
& b_{12}=\frac{\alpha_{7} k_{0} \beta T_{0} \omega^{*}}{\sigma_{0} \mu_{0}^{2} H_{0}^{2}}, b_{13}=\epsilon_{0} \mu_{0} i \xi+\frac{\sigma_{0} \mu_{0}}{\omega^{*}}, O_{1_{n}}=\frac{m_{6}}{\left(L_{13}-\alpha_{n}^{2}\right)}, \\
& \alpha_{9}=\sqrt{a^{2}-\epsilon_{0} \xi^{2}}, \quad b_{14}=\left[L _ { 5 } ( \alpha _ { n } ^ { 2 } - a ^ { 2 } ) \left(L _ { 8 } \left(m_{20} \alpha_{n}^{2}-\right.\right.\right. \\
& \left.\left.L_{14}\right)-m_{12} L_{15}\left(\alpha_{n}^{2}-a^{2}\right)\right)+m_{11}\left(\alpha_{n}^{2}-a^{2}\right)\left(\left(m_{20} \alpha_{n}^{2}-\right.\right. \\
& \left.\left.\left.L_{14}\right)\left(\alpha_{n}^{2}-L_{3}\right)+L_{4} L_{15}\left(\alpha_{n}^{2}-a^{2}\right)\right)\right], \quad b_{15}=\left[L _ { 5 } \left(\alpha_{n}^{2}-\right.\right. \\
& \left.a^{2}\right)\left(\left(\alpha_{n}^{2}-L_{7}\right)\left(m_{20} \alpha_{n}^{2}-L_{14}\right)+m_{12} L_{16}\left(\alpha_{n}^{2}-a^{2}\right)\right)+ \\
& \left.m_{11}\left(\alpha_{n}^{2}-a^{2}\right)\left(\left(m_{20} \alpha_{n}^{2}-L_{14}\right) L_{6}-L_{4} L_{16}\left(\alpha_{n}^{2}-a^{2}\right)\right)\right], \\
& O_{2_{n}}=H_{1_{n}}=\frac{b_{14}}{b_{15}}, O_{3_{n}}=\frac{\left(\alpha_{n}^{2}-L_{1}\right)\left(L_{11}-L_{10} O_{2 n}\right)}{\left(\alpha_{n}^{2}-L_{1}\right)\left(\alpha_{n}^{2}-L_{9}\right)+L_{2} L_{12}\left(\alpha_{n}^{2}-a^{2}\right)}, \\
& O_{4_{n}}=\frac{-L_{2}\left(\alpha_{n}^{2}-a^{2}\right) O_{3 n}}{\left(\alpha_{n}^{2}-L_{1}\right)}, \quad O_{5_{n}}=\frac{\left(L_{15}-L_{16} O_{2 n}\right)}{\left(m_{20} \alpha_{n}^{2}-L_{14}\right)}, \quad O_{6_{n}}=i a O_{3_{n}} \\
& \alpha_{n}, O_{7_{n}}=-i a-\alpha_{n} O_{3_{n}}, O_{8_{n}}=\operatorname{iam}_{29} O_{6_{n}}-\alpha_{n} m_{30} O_{7_{n}}+ \\
& m_{31} O_{2_{n}}-m_{32} O_{1_{n}}, \quad O_{9_{n}}=\operatorname{iam}_{30} O_{6_{n}}-\alpha_{n} m_{29} O_{7_{n}}+ \\
& m_{31} O_{2_{n}}-m_{32} O_{1_{n}}, O_{10_{n}}=\operatorname{iam}_{33} O_{7_{n}}-\alpha_{n} m_{33} O_{6_{n}} \text {, } \\
& O_{11_{n}}=a^{2} b_{6} O_{5_{n}}-\alpha_{n}{ }^{2} b_{7} O_{5_{n}}, \quad O_{12_{n}}=i a\left(b_{8}+b_{9}\right) \alpha_{n} O_{5_{n}} \text {, } \\
& O_{13_{n}}=\frac{o_{4 n}+i \xi c_{1}^{2} o_{6 n}+b_{12} O_{1 n}}{b_{13}}, H_{3_{n}}=\frac{-L_{2}\left(\alpha_{n}^{2}-a^{2}\right) H_{3 n}}{\left(\alpha_{n}^{2}-L_{1}\right)}, \\
& H_{2_{n}}=\frac{\left(\alpha_{n}^{2}-L_{1}\right)\left(L_{11}-L_{10} H_{2 n}\right)}{\left(\alpha_{n}^{2}-L_{1}\right)\left(\alpha_{n}^{2}-L_{9}\right)+L_{2} L_{12}\left(\alpha_{n}^{2}-a^{2}\right)}, H_{4_{n}}=\frac{\left(L_{15}-L_{16} H_{2 n}\right)}{\left(m_{20} \alpha_{n}^{2}-L_{14}\right)}
\end{aligned}
$$

\section{References:}

[1] M. A. Biot, "Non-linear theory of elasticity and the linearized case for a body under initial stress," The London, Edinburgh, and Dublin Philosophical Magazine and Journal of Science, 27(183), 468-489, 1939.

[2] H. Lord and Y. Shulman, "A generalized dynamical theory of thermoelasticity," Journal of the Mechanics and Physics of Solids, 15(5), 299-309, 1967.

[3] E. Green and K. A. Lindsay, “Thermoelasticity,” Journal of Elasticity, 1972.

[4] V. K. Agarwal, "On electromagneto-thermoelastic plane waves," Acta Mechanica, 34(3), 181-191, 1979.

[5] G. Paria, "Magneto-elasticity and magnetothermoelasticity," In Advances in Applied Mechanics. Elsevier, 73-112, 1966.

[6] H. Youssef, "Generalized magneto-thermoelasticity in a conducting medium with variable material properties," Applied Mathematics and Computation, 173(2), 822833, 2006.

[7] R. A. Grot, "Thermodynamics of a continuum with microstructure," International Journal of Engineering Science, 7(8), 801-814, 1969.

[8] P. Riha, "On the microcontinuum model of heat conduction in materials with inner structure," International Journal of Engineering Science, 14(6), 529-535, 1976.

[9] P. S. Casas and R. Quintanilla, "Exponential stability in thermoelasticity with microtemperatures," International Journal of Engineering Science, 43(1), 33-47, 2005.

[10] R. Quintanilla and D. Iesan, "On the theory of thermoelasticity with microtemperatures," Journal of Thermal Stresses, 23(3), 199-215, 2000.

[11] D. Iesan, "On a theory of micromorphic elastic solids with microtemperatures," Journal of Thermal Stresses, 24(8), 737-752, 2001.

[12] M. Svanadze, "Fundamental solutions of the equations of the theory of thermoelasticity with microtemperatures," Journal of Thermal Stresses, 27(2), 151-170, 2004.

[13] M. I. Othman and E. M. Abd-Elaziz, "Influence of gravity and microtemperatures on the thermoelastic porous medium under three theories," International Journal of Numerical Methods for Heat \& Fluid Flow, 29(9), 3242-3262, 2019.

[14] R. Quintanilla, "Uniqueness in thermoelasticity of porous media with microtemperatures," Archives of Mechanics, 61(5), 371-382, 2009.

[15] C. Eringen, "Microcontinuum field theories: I. Foundations and solids," Springer Science \& Business Media, 2012.

[16] C. Eringen and E. S. Suhubi, "Nonlinear theory of simple microelastic solids," International Journal of Engineering Science, 2(2), 189-203, 1964.

[17] K. K. Kalkal, R. Goyal, and S. Deswal, "Thermomechanical interactions in a magneto Thermoelastic solid with microtemperatures and diffusion," Microsystem Technologies, 25(10), 3747 3763, 2018.

[18] S. C. Cowin and J. W. Nunziato, "Linear elastic materials with voids," Journal of Elasticity, 13(2), 125$147,1983$.

[19] J. W. Nunziato and S. C. Cowin, "A nonlinear theory of elastic materials with voids," Archive for Rational Mechanics and Analysis, 72 (2), 175-201, 1979.

[20] D. Iesan, "A theory of thermoelastic materials with voids," Acta Mechanica, 60(1), 67-89, 1986.

[21] Pompei and A. Scalia, "On the asymptotic spatial behavior in linear thermoelasticity of materials with voids," Journal of Thermal Stresses, 25(2), 183-193, 2002.

[22] S. Chirita and A. Scalia, "On the spatial and temporal behavior in linear thermoelasticity of materials with voids," Journal of Thermal Stresses, 24(5), 433-455, 
2001.

[23] M. I. A. Othman, M. E. Zidan, and M. I. Hilal, "Effect of magnetic field on a rotating thermoelastic medium with voids under thermal loading due to laser pulse with energy dissipation," Canadian Journal of Physics, 13591371, 2014.

[24] D. Iesan, "A theory of initially stressed thermoelastic material with voids," An. Stiint. Univ. Ai. I. Cuza Lasi Sect. I a Mat, 33, 167-184, 1987.

[25] M. A. Goodman and S. C. Cowin, "A continuum Theory for granular materials," Archive for Rational Mechanics and Analysis, 44(4), 249-266, 1972.

[26] J. Jaric, "Theory of thermoelasticity of granular materials," Rev. Roum. Sci. Techn., Méc. Appl 24, 793$805,1979$.

[27] M. I. A. Othman, R. S. Tantawi, and E. M. Abd-Elaziz, "Effect of initial stress on a thermoelastic medium with voids and microtemperatures," Journal of Porous Media, 19(2), 155-172, 2016

[28] K. K. Kalkal, R. Kumar, A. Gunghas, and S. Deswal, "Wave propagation in an initially stressed magnetothermoelastic medium with voids and microtemperatures," Journal of Thermal Stresses, 43(8), 962-980, 2020.

[29] M. I. A. Othman and E. M. Abd-Elaziz, "Effect of initial stress and hall current on a magneto-thermoelastic porous medium with microtemperatures," Indian Journal of Physics, 93(4), 475-485, 2018.

[30] M. I. A. Othman and E. M. Abd-Elaziz, "Plane waves in A magneto-thermoelastic solids with voids and microtemperatures due to hall current and rotation," Results in Physics, 7, 4253-4263, 2017.

[31] K. Ames and B. Straughan, "Continuous dependence results for initially prestressed thermoelastic bodies," International Journal of Engineering Science, 30(1), 713, 1992.

[32] Montanaro, "On singular surfaces in isotropic linear thermoelasticity with initial stress," The Journal of the Acoustical Society of America, 106(3), 1586-1588, 1999.

[33] Abbas and M. I. A. Othman, "Generalized thermoelastic interaction in a fiber-reinforced anisotropic half space under hydrostatic initial stress," Journal of Vibration and Control, 18(2), 175-182, 2011.

[34] M. A. Ezzat and M. Z. A. Elall, "Generalized magneto thermoelasticity with modified ohm's law," Mechanics of Advanced Materials and Structures, 17(1), 74-84, 2009.

[35] N. Sarkar, "Generalized magneto $\neg$ thermoelasticity with modified ohm's law under three theories," Computational Mathematics and Modeling, 25(4), 544564, 2014. 